\title{
The Big Bang May Had Never Existed
}

\author{
Salah A. Mabkhout \\ Department of Mathematics-Faculty of Education-Thamar University \\ Thamar- Republic of Yemen
}

\begin{abstract}
The main pillar of the Big Bang paradigm is the expansion of the Universe predicted by the cosmological redshift. Singularity is inevitable in the Big Bang model. The Universe is hyperbolic as we did prove mathematically; where the cosmological redshift is no longer a distance indicator. After all, in the hyperbolic spacetime a group of objects would grow apart even when not moving as their worldlines would be divergent. We show the manifold of the hyperbolic Universe is complete with no singular points. While the distance horizon in the Big Bang flat spacetime is finite, the distance horizon is infinite in the hyperbolic universe. The pillars of the big Bang and its consequences had been refuted and disproved or reinterpreted.
\end{abstract}

Keywords: Big Bang, expanding universe, static universe, time dilation, distance horizon, nonsingularity.

\section{Introduction}

The Universe is not flat. We did prove that the universe globally is hyperbolic. We exhibit the hyperbolic structure of the universe where the curvature manifests itself as a cosmological redshift. Newton first law states that the body keeps moving with a uniform velocity in straight line. Similarly, the free fall of an object in a flat spacetime is uniform. An accelerated motion is described by a curve. For large structure, the curvature of the spacetime can't be ignored. The distant objectse.g. supernovae - were influenced under the curvature of the spacetime. They possess an accelerating free fall due to the curvature of the hyperbolic spacetime that manifests itself by the equation of the state $p=-r$, which is the property of the hyperbolic structure of the Universe. The increases in the redshift for distant objects reflect the increases in the curvature. It doesn't indicate an accelerating expansion of the universe. After all, in the hyperbolic spacetime a group of objects would grow apart even when not moving as their worldlines would be divergent; this we mean by the expansion of the hyperbolic spacetime. The distance horizon is infinite in a hyperbolic universe, which answers the question: Where does the universe expand into? The observable Universe is expanding into a pre-existing infinite distance horizon. This question is forbidden and prohibited question in the Standard Big Bang Cosmology. The Big Bang theorists insist that the above question has no meaning, instead of saying that they have no answer or that it violates their entire paradigm. We remove the singularity from the mathematical model, represented by the General Relativity Theory and the hyperbolic spacetime. .

\section{The Hyperbolic Spacetime}

To obtain the dynamical equation of cosmology, we should combine Einstein field equations

$$
R_{m n}-\frac{1}{2} g_{m n} R=8 p G T_{m n}
$$

with the isotropic homogeneous Robertson- Walker's line-element:

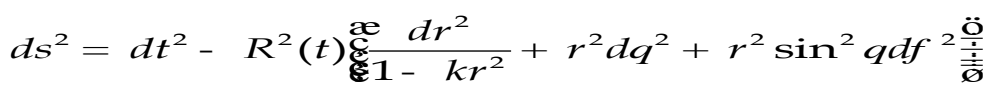

to get Friedmann`s equations: 
$\dot{R}^{2}+k=(8 p / 3) r R^{2}$

$2 R \ddot{R}+\dot{R}^{2}+k=-8 p p R^{2}$

Where $p$ is the pressure and $\rho$ is the energy density of the cosmological fluid and $k$ is the curvature.

Method of solution:[1]

(i) Now we shall solve the differential equation (1) by separating the variables. We assume the Big Bang Model as an initial condition (i.e. $R=0$ when $t=0$ ).

$$
\begin{aligned}
& \dot{R}^{2}+k=(8 p / 3) r R^{2} \\
& \dot{R}^{2}=(8 p / 3) r R^{2}-k \\
& \dot{R}=\sqrt{\left({ }^{8 p r} / 3\right) R^{2}-k} \\
& d R / \sqrt{\left({ }^{8 p r} / 3\right) R^{2}-k}=d t \\
& d R / \sqrt{R^{2}-3 k / 8 p r}=\sqrt{8 p r / 3} d t
\end{aligned}
$$

Differential equation (1) allows one to deal with $\rho_{\mathrm{j}}$ as a parameter since it's not an explicit function of $t$, so Eq. (1) can be solved for any chosen fixed value, $\rho$ from the stream of the various values of the parameter $\rho$ : .

$$
r_{1}, r_{2}, \ldots, r_{\text {planck }}, \ldots, r_{j}, \ldots, r_{\text {now }}
$$

By means of the mean value theorem, we assume approximately that $\rho_{j}$ evolves to the fixed physical value $\rho_{j}$ exactly simultaneously associated to the state $\left(\mathrm{t}_{\mathrm{j}}, \mathrm{R}_{\mathrm{j}}\right)$ since $\rho_{\mathrm{j}}$ is not defined and not continuous at the point of singularity $t=0$, put

$$
r(\mathrm{c})=r_{\mathrm{j}}: 0<\mathrm{c} £ \mathrm{t}
$$

$\hat{\mathrm{O}}_{\mathrm{o}}^{R} d r / \sqrt{r^{2}-3 k / 8 p r_{\mathrm{j}}}=\sqrt{8 p r_{\mathrm{j}} / 3}{\underset{\mathrm{o}}{\mathrm{o}}}^{t} d t$

$\cosh ^{-1}\left(R / \sqrt{3 k / 8 p r_{\mathrm{j}}}\right)-\cosh ^{-1} \mathrm{O}=t \sqrt{8 p r_{\mathrm{j}} / 3}$ analysis as follows .

$$
\begin{aligned}
& \cosh ^{-1} x=\ln \left(x \pm \sqrt{x^{2}-1}\right) \\
& \cosh ^{-1} 0=\ln \pm \sqrt{-1}=\ln \sqrt{-1}, \text { or }, \cosh ^{-1} 0=\ln -\sqrt{-1} \\
& \cosh ^{-1} 0=(1 / 2) \ln (-1), \text { or }, \cosh ^{-1} 0=\ln (-1)+(1 / 2) \ln (-1) \\
& \because e^{i p}=-1 \\
& \backslash \ln (-1)=i p \\
& \backslash \cosh ^{-1} 0=i p / 2, \text { or } \\
& \cosh ^{-1} 0=3 i p / 2
\end{aligned}
$$


Substitute the first value $\cosh ^{-1} 0=i p / 2$ in equation (3), we get:

$$
\begin{aligned}
& R(t)=\sqrt{3 k / 8 p r_{\mathrm{j}}} \cdot \cosh \left(t \sqrt{8 p r_{\mathrm{j}} / 3}+p i / 2\right) \\
& R(t)=\sqrt{3 k / 8 p r_{\mathrm{j}}} \cdot\left(\cosh t \sqrt{8 p r_{\mathrm{j}} / 3} \cdot \cosh (p i / 2)+\sinh (p i / 2) \cdot \sinh t \sqrt{8 p r_{\mathrm{j}} / 3}\right) \\
& R(t)=\sqrt{3 k / 8 p r_{\mathrm{j}}} \cdot\left(\cosh t \sqrt{8 p r_{\mathrm{j}} / 3} \cdot \cos (p / 2)+i \sin (p / 2) \cdot \sinh t \sqrt{8 p r_{\mathrm{j}} / 3}\right) \\
& R(t)=i \sqrt{3 k / 8 p r_{\mathrm{j}}} \cdot \sinh t \sqrt{8 p r_{\mathrm{j}} / 3}
\end{aligned}
$$

Since the function $r(t)$ is always positive, so is any chosen fixed value $r{ }_{j}$. A simple analysis shows that the $\mathrm{R}(\mathrm{t})$ scale solution represented in the last equation is complex if $k$ is positive, negative if $k$ is negative and vanishes if $k$ is zero. So the first value $\cosh ^{-1} 0=i p / 2$ is rejected. Substitute the other value $\cosh ^{-1} 0=3 i p / 2$ in equation (3), we get

$$
\begin{aligned}
& R(t)=\sqrt{3 k / 8 p r_{\mathrm{j}}} \cdot \cosh \left(t \sqrt{8 p r_{\mathrm{j}} / 3}+3 p i / 2\right) \\
& \left.R(t)=\sqrt{3 k / 8 p r_{\mathrm{j}}} \cdot \cosh t \sqrt{8 p r_{\mathrm{j}} / 3} \cdot \cosh (3 p i / 2)+\sinh (3 p i / 2) \sinh t \sqrt{8 p r_{\mathrm{j}} / 3}\right) \\
& R(t)=\sqrt{3 k / 8 p r_{\mathrm{j}}}\left(\cosh t \sqrt{8 p r_{\mathrm{j}} / 3} \cdot \cos (3 p / 2)+i \sin (3 p / 2) \sinh t \sqrt{8 p r_{\mathrm{j}} / 3}\right) \\
& R(t)=-i \sqrt{3 k / 8 p r_{\mathrm{j}}} \sinh t \sqrt{8 p r_{\mathrm{j}} / 3}
\end{aligned}
$$

The $R(t)$ scale solution in the last equation is real, positive and non-vanishing if and only if $k$ is negative. Since $k$ is

$$
\begin{aligned}
& \text { normalized, } \quad \text { substitute } \quad \mathrm{k}=-1, \quad \text { in } \quad \text { the } \\
& R(t)=-i \sqrt{3 k / 8 p r_{\mathrm{j}}} \sinh t \sqrt{8 p r_{\mathrm{j}} / 3} \\
& R(t)=-i \sqrt{-3 / 8 p r_{\mathrm{j}}} \sinh t \sqrt{8 p r_{\mathrm{j}} / 3} \\
& R(t)=-i . i \sqrt{3 / 8 p r_{\mathrm{j}}} \sinh t \sqrt{8 p r_{\mathrm{j}} / 3} \\
& R(t)=\sqrt{3 / 8 p r_{\mathrm{j}}} \sinh t \sqrt{8 p r_{\mathrm{j}} / 3}
\end{aligned}
$$$$
\text { last equation, we get: }
$$
get:

Which mean that $R(t)$ either vanishes if $k=0$ or complex if $k=1$.Thus, the curvature $k$ must be negative and consequently the universe must be hyperbolic and open. Note that the solution represented by Eq. (4) is evaluated only for the values simultaneously associated with $r_{j}$, namely $\left(\boldsymbol{R}_{j}, \boldsymbol{t}_{j}\right)$

$$
R_{j}=\sqrt{3 / 8 p r_{j}} \sinh t_{j} \sqrt{8 p r_{j} / 3}
$$

\section{Verification Of the Time Evolution Equation of the Universe \\ (i) Planck scale:[4]}


The time evolution equation of the universe successfully predicts the Planck length at micro-cosmos scale as well as it predicts the current observed large structure at macro-cosmos scale.

$1 \mathrm{sec}=2.997 \times 10^{10} \mathrm{~cm}$

Planck length $=L_{p}=\sqrt{G h / c^{3}}=1.6^{\prime} 10^{-33} \mathrm{~cm}$

Planck time $=t_{p}=L_{p} / c=\sqrt{G h / c^{5}}=5.4^{\prime} 10^{-44} s$

Planck density $==3.8789 \times 10^{62} \mathrm{~cm}$

Substitute the above data in the time evolution equation of the universe at Planck scale

$$
\begin{aligned}
& R_{p}=\sqrt{3 / 8 p r_{p}} \sinh t_{p} \sqrt{8 p r_{p} / 3} \\
& R_{p}=\sqrt{3 / 8 p^{\prime} 3.8789^{\prime} 10^{62}} \sinh \sqrt{8 p^{\prime} 3.8789^{\prime} 10^{62} / 3^{\prime}} 5.4^{\prime} 10^{-44} 2.997^{\prime} 10^{10} \\
& =0.175423 \times 10^{-31} \times \sinh 0.092255888 \\
& =0.175423 \times 10^{-31} \times 0.092386811 \\
& =1.62 \times 10^{-33} \mathrm{~cm}=\mathrm{L}_{p}=\text { Planck length. Hence }
\end{aligned}
$$$$
R_{p}=\sqrt{3 / 8 p r_{p}} \sinh \sqrt{8 p r_{p} / 3} t_{p}=L_{p}=\sqrt{G \hbar / c^{3}}
$$

(ii) Current scale:

The energy density now $r_{\text {now }}=10^{-31} \mathrm{~g} / \mathrm{cm}^{3}=7.425 \times 10^{-60} \mathrm{~cm}^{-2}$

The age of the Universe (approximately) $t_{\text {now }}=13.7 \times 10^{9} \mathrm{yr}=1.2974585^{\prime} 10^{28} \mathrm{~cm}$

Substitute the above data in the hyperbolic time evolution equation of the Universe, yields, .

$$
\begin{aligned}
& R_{j}=\sqrt{3 / 8 p r_{j}} \sinh \text { é }_{j} \sqrt{8 p r_{j} / 3} \text { ù }
\end{aligned}
$$

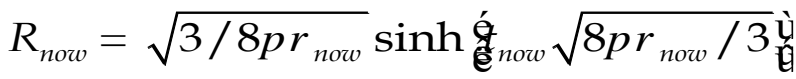

$$
\begin{aligned}
& R_{\text {now }}=\sqrt{3 /\left(8 p^{\prime} 7.425^{\prime} 10^{-60}\right)^{\prime}} \\
& \text { sinh 重.2974585' } 10^{28} \cdot \sqrt{8 p^{\prime} 7.425^{\prime} 10^{-60} / 3} \text { 稁 } \\
& R_{\text {now }}=1.6^{\prime} 10^{29} \cdot \sinh 0.08287 \\
& R_{\text {now }}=1.3^{\prime} 10^{28} \mathrm{~cm}
\end{aligned}
$$

\section{The increase in the cosmological redshift accounts for the increases in the curvature, not for the accelerated} expansion.

We shall see that the solution of equation (1) satisfies the second order differential equation (2) in order to be consistent. We have from the solution of Eq. (1) for any chosen value $\rho_{j}$ 


$$
\begin{aligned}
& R=\sqrt{\frac{3}{8 p r_{j}}} \sinh t \sqrt{\frac{8 p r_{j}}{3}} \\
& \dot{R}=\operatorname{cosht} \sqrt{\frac{8 p r_{j}}{3}} \\
& \dot{R}=\sqrt{\frac{8 p r_{j}}{3}} \sinh t \sqrt{\frac{8 p r_{j}}{3}}=\frac{8 p r_{j}}{3} R
\end{aligned}
$$

Substitute these values in Eq. (2), and $k=-1$, yields,

$$
\begin{aligned}
& 2 R \ddot{R}+\dot{R}^{2}-1=-8 p p R^{2} \\
& 2 R \underset{\frac{\mathrm{g}}{\mathrm{E}} 8 p r_{j}}{3} R \frac{\ddot{\mathrm{O}}}{\dot{\overline{\mathrm{I}}}}+\cosh ^{2} \sqrt{\frac{8 p r_{j}}{3}}-1=-8 p p R^{2} \\
& 2 R^{2} \frac{28 p r_{j}}{3} \frac{\ddot{\circ}}{\dot{\bar{\Xi}}}+\sinh ^{2} \sqrt{\frac{8 p r_{j}}{3}}=-8 p p R^{2}
\end{aligned}
$$

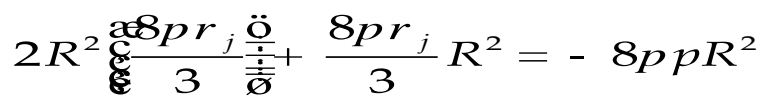

$$
\begin{aligned}
& 8 p r_{j} R^{2}=-8 p p R^{2} \\
& p=-r_{j}
\end{aligned}
$$

The last equation is known as the equation of state of cosmology. The argument of the solution predicts the equation of state of cosmology $p=-r_{j}$. Since the energy density is always positive, the negative pressure implies an accelerated expansion of the universe. Hence equations (1) and (2) are consistent for any chosen fixed value $\boldsymbol{r} j$ of the parameter $r$. The argument of the solution predicts the equation of state $p=-r_{j}$. We exhibit the hyperbolic structure of the universe that explains the accelerating expansion of the universe without needs for additional components, dark energy. One explanation for dark energy is that it is a property of space. The simplest explanation for dark energy is that it is simply the "cost of having space": that is, a volume of space has some intrinsic, fundamental energy. Just the ordinary energy density state $r_{j}$ remains in the Hyperbolic Universe to derive the accelerating expansion equivalent to its negative pressure. Hyperbolic Universe involves zero cosmological constant (the vacuum energy) [2]. The negative pressure $p=-r_{j}$ is the property of the hyperbolic structure of the Universe. Flat universe dominated by matter is modeled as a zero pressure-dust universe model. The expansion of the universe would be slowing due to the gravity attraction, which is incorrect as we shall see below: instein postulates [3] that the matter dominated universe could be modeled as dust with zero pressure in order to simplify and solves Friedmann's equations : .

$$
\begin{aligned}
& \dot{R}^{2}+k=(8 p / 3) r R^{2} \\
& 2 R \ddot{R}+\dot{R}^{2}+k=0 \\
& 2 \ddot{R} / R+(8 p / 3) r=0 \\
& \ddot{R}=-(8 p / 3) r R / 2<0 \\
& \backslash \ddot{R}<0
\end{aligned}
$$


The pressure less form of Eq. (2) describes a decelerating expansion state of the universe which is described by the energy tensor of matter for dust where $p=0$. We solved the second dynamical equation of cosmology, the space-space component; in it is pressure less form:

$$
\begin{aligned}
& 2 R \ddot{R}+\dot{R}^{2}+k=0 \\
& \because k=-1 \\
& 2 R \ddot{R}+\dot{R}^{2}-1=0
\end{aligned}
$$

to be $t=R$, which satisfies the last differential equation. Substitute $t=R, k=-\mathbf{I}_{\text {in }}$ the first dynamical equation (2) .

$$
\begin{aligned}
& \dot{R}^{2}+k=(8 p / 3) r R^{2} \\
& (1)^{2}-1=(8 p / 3) r R^{2} \\
& \mathrm{O}=(8 p / 3) r R^{2} \\
& \backslash r=0
\end{aligned}
$$

Hence the zero pressure does not lead to a dusty universe. In fact zero pressure Universe is an empty space, since $r=\mathbf{O}$. In the presence of pressure, from Eqs (2) and (1) we can obtain

$$
\begin{aligned}
& \ddot{R}=-\frac{4 p}{3}(r+3 p) R \\
& \because p=-r \\
& \backslash \ddot{R}=\frac{8 p}{3} R>0
\end{aligned}
$$

which guarantees an accelerating expansion of the universe.

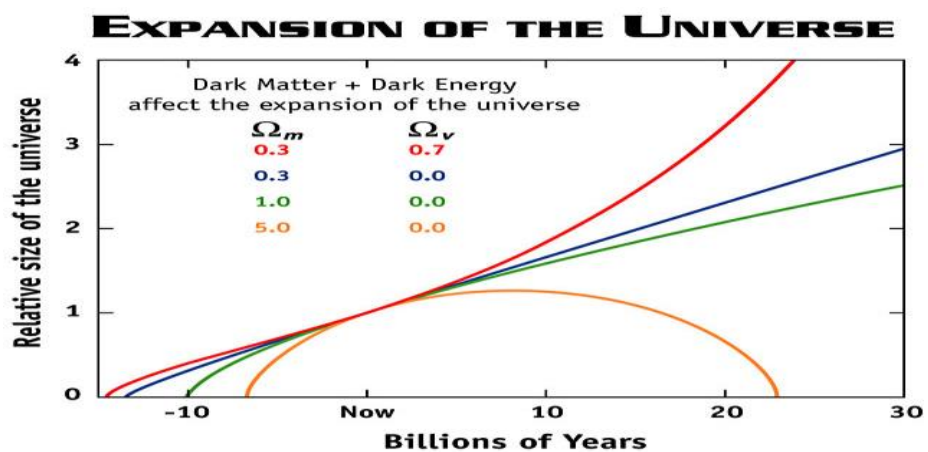

Figure 1. Scientists used to think that the universe was described by the yellow, green, or blue curves. But surprise, it's actually the red curve instead.

Newton first law states that the body keeps moving with a uniform velocity in straight line. Similarly, the free fall of an object in a flat spacetime is uniform. An accelerated motion is described by a curve. For large structure, the curvature of the spacetime can't be ignored. It is clear from Fig (1) the expansion of the universe is described by a hyperbolic curve. The distant objects- e.g. supernovae - were influenced under the curvature of the spacetime. They possess an accelerating free fall due to the curvature of the hyperbolic spacetime that manifests itself by an equation of a state $p=-r$, which is the property of the hyperbolic structure of the Universe. The Universe is not flat. We did prove that, the universe globally is hyperbolic. We exhibit the hyperbolic structure of the universe where the curvature manifests itself as a cosmological 
redshift. The increases in the redshift for distant objects reflect the increases in the curvature. It doesn't indicate an accelerating expansion of the universe.

\section{Nonsingular Big Bang.}

An initial-value problem: Given the state of a system at some moment in time, what will be the state at some later time? Future events can be understood as consequences of initial conditions plus the laws of physics. Could the dynamical nature of the spacetime background break down an initial-value formulation in general relativity? In general relativity, a singularity is a place that objects or light rays can reach in a finite time where the curvature becomes infinite, or spacetime stops being a manifold. Singularities can be found in all cosmological solutions which don't have scalar field energy or a cosmological constant. Curvature is associated with gravity and hence curvature singularities correspond to "infinitely strong gravity." There are several possibilities of how such infinitely strong gravity can manifest itself. For instance, it could be that the energy density becomes infinitely large - this is called a "Ricci singularity", As an example of a Ricci singularity, the evolution of energy density in a universe described by a big bang model. As you go towards the left - corresponding to earlier and earlier instances of cosmic time zero - the density grows beyond all bounds and at cosmic time zero - at the big bang - it was infinitely high. A path in spacetime is a continuous chain of events through space and time [5]. While there are competing definitions of spacetime singularities, the most central, and widely accepted, criterion rests on the possibility that some spacetimes contain incomplete paths. Indeed, the rival definitions (in terms of missing points or curvature pathology) still make use of the notion of path incompleteness. While path incompleteness seems to capture an important aspect of the intuitive picture of singular structure, it completely ignores another seemingly integral aspect of it: curvature pathology. If there are incomplete paths in a spacetime, it seems that there should be a reason that the path cannot go farther. The most obvious candidate explanation of this sort is something going wrong with the dynamical structure of the spacetime, which is to say, with the curvature of the spacetime. This suggestion is bolstered by the fact that local measures of curvature do in fact blow up as one approaches the singularity of a standard black hole or the big bang singularity. However, there is one problem with this line of thought: no species of curvature pathology we know how to define is either necessary or sufficient for the existence of incomplete paths. "At the heart of all of our conceptions of a spacetime singularity is the notion of some sort of failing: a path that disappears, points that are torn out, spacetime curvature that becomes pathological. However, perhaps the failing lies not in the spacetime of the actual world, but rather in the theoretical description of the spacetime" [5]. That is, perhaps we shouldn't think that general relativity is accurately describing the world when it posits singular structure! Indeed, in most scientific arenas, singular behavior is viewed as an indication that the theory being used is deficient. It is therefore common to claim that general relativity, in predicting that spacetime is singular, is predicting its own demise, and that classical descriptions of space and time break down at black hole singularities and at the Big Bang. Such a view seems to deny that singularities are real features of the actual world, and to assert that they are instead merely artifices of our current (flawed) physical theories. Many physicists and philosophers resist that singularities are real. Some argue that singularities are too repugnant to be real. Others argue that the singular behavior at the center of black holes and at the beginning of time points to the limit of the domain of applicability of general relativity. Note that the hyperbolic universe inflates exponentially produces an accelerated expansion of the universe without cosmological constant or scalar field. We have shown [S. A. Mabkhout, Phys. Essays 26,422 (2013)] that general relativity doesn't break down at large cosmological scale since it predicts both the accelerated expansion of the universe (without invoking dark energy) and predicts the galaxy flat rotation curve (without invoking dark matter) [6]. General relativity didn't break down at Planck scale as we had shown [S. A. Mabkhout, Phys. Essays 25, 112 (2012)] [1]. In this research we shall prove that the time evolution equation of the universe characteristics the hyperbolic universe and traces its manifold dynamical geometry shouldn't break down even at the initial Big Bang moment. Our task is to remove the singularity from the mathematical model, represented by the General Relativity Theory and the hyperbolic spacetime, underlying the Big Bang Theory. Our main point is to examine whether the state point:

$$
\left(R_{B . B}, r_{B . B}, t_{B . B}\right)=(\mathrm{O}, ¥, \mathrm{O})
$$

constitutes a singular point in the manifold? Is it really a missing point of the manifold? Does the local measure of curvature blow up as one approach this point? Does the density grow beyond all bounds, infinitely high as one approach this point? Our hyperbolic universe is a manifold weaved by the time evolution equation of the universe since the Big Bang

$$
R_{j}=\sqrt{3 / 8 p r_{j}} \sinh \text { é }_{j} \sqrt{8 p r_{j} / 3} \text { ùu }
$$


Which reflects the structure of the manifold whether it possesses a singular point or not? If we assume the density $\boldsymbol{r}_{j}$ and the time $t_{j}$ runs independently from each other, we may evaluate the limit at the Big Bang

$$
\begin{aligned}
& \left(r_{j}, t_{j}\right)=\left(r_{B B}, t_{B B}\right) \mathbb{R}(¥, 0)
\end{aligned}
$$

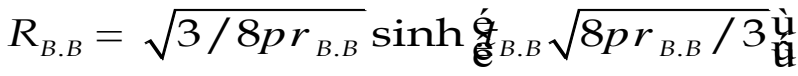

$$
\begin{aligned}
& R_{B . B}=\lim _{\left(r_{B . B}, t_{B . B}\right) \mathbb{B}(¥, 0)} \sqrt{3 / 8 p r_{B . B}} \sinh \dot{f}_{B . B} \sqrt{8 p r_{B . B} / 3}
\end{aligned}
$$

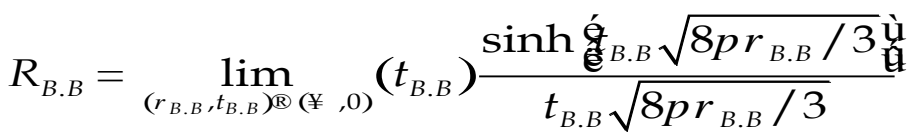

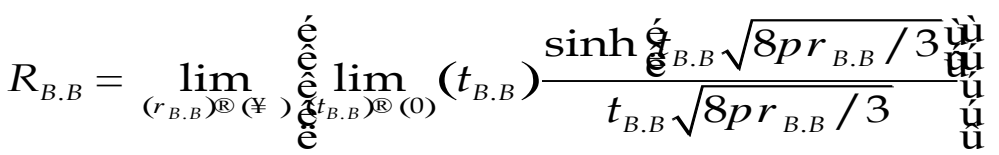

$$
\begin{aligned}
& R_{B . B}=\lim _{\left(r_{B . B}\right) \mathbb{R}(¥)}\left[0^{\prime} 1\right]=0
\end{aligned}
$$

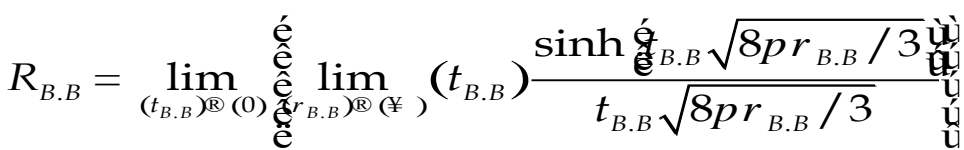

$$
\begin{aligned}
& R_{B . B}=\lim _{\left(t_{B . B}\right) \mathbb{R}(0)}[¥]=¥
\end{aligned}
$$

The limit does not exist since it is not unique. Let us treat the limit from a different point of view, namely the dependent evolution for both the density $r_{j}$ and the time $t_{j}$. Now we are interesting to explore how both the density $\boldsymbol{r}_{j}$ and the time $\boldsymbol{t}_{j}$ are dependently evolved? Consider the factor $t_{j} \sqrt{r_{j}}$ appears in the time evolution equation of the Universe. Calculate the value of $t_{j} \sqrt{r_{j}}$ at the given two well known sets of data, namely the Planck scale and the current scale:

$$
\begin{aligned}
& t_{p} \sqrt{r_{p}}=5.4^{\prime} 10^{-44}{ }^{\prime} 2.997^{\prime} 10^{10} \sqrt{3.8789^{\prime} 10^{62}}=0.032 \\
& t_{\text {now }} \sqrt{r_{\text {now }}}=1.2974585^{\prime} 10^{28} \cdot \sqrt{7.425^{\prime} 10^{-60}}=0.034
\end{aligned}
$$

The two values are approximately equal no matter how large the difference between the two states, which is of order $10^{61}$ . Hence it is very reasonable that $t_{j} \sqrt{r_{j}}$ remains approximately constant through the whole evolution of the cosmos, even at the Big Bang. The infinitely large density is struggled by the infinitesimally small time and vice versa, in our mathematical model. This process prevents the scale factor from blows up by the infinitely large density. Since the data at Planck scale is accurate, we assume $t_{j} \sqrt{r_{j}}=0.032$. Hence 


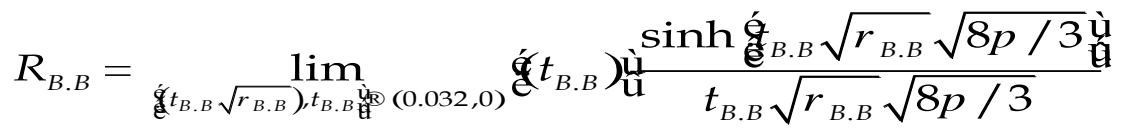

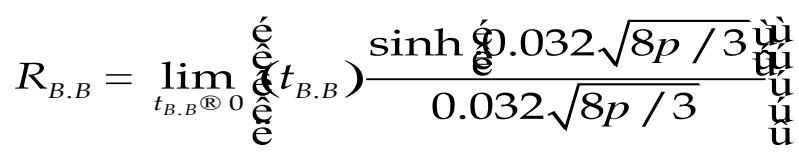

$$
\begin{aligned}
& R_{B . B}=\lim _{t_{B . B}(\mathcal{B}}\left(t_{B . B}\right)^{\prime} 1.00187 \stackrel{\grave{u}}{\mathrm{u}}=0
\end{aligned}
$$

The limit exists. The manifold consists of its limiting point and hence it is complete. Thus there exists a continuous path governs the time evolution of the Universe since the Big Bang. Hence the Big Bang is nonsingular.

\section{Cosmological Redshift}

In the early part of the twentieth century, Slipher, Hubble and others made the first measurements of the redshifts and blueshifts of galaxies beyond the Milky Way. They initially interpreted these redshifts and blueshifts as due solely to the Doppler Effect, but later Hubble discovered a rough correlation between the increasing redshifts and the increasing distance of galaxies. Theorists almost immediately realized that these observations could be explained by a different mechanism for producing redshifts. Hubble's law of the correlation between redshifts and distances is required by models of cosmology derived from general relativity that have a metric expansion of space. As a result, photons propagating through the expanding space are stretched, creating the Cosmological redshift. The Cosmological redshift differs from the Doppler Effect redshifts described above, because the velocity boost (i.e. the Lorentz transformation) between the source and observer is not due to classical momentum and energy transfer, but instead the photons increase in wavelength and redshift as the space through which they are travelling expands. This effect is prescribed by the current cosmological model as an observable manifestation of the time-dependent cosmic scale factor (a) in the following way: [7]

$$
1+z=\frac{a_{\text {now }}}{a_{\text {then }}} .
$$

This type of redshift is called the Cosmological redshift or Hubble redshift. These galaxies are not receding simply by means of a physical velocity in the direction away from the observer; instead, the intervening space is stretching, which accounts for the large-scale isotropy of the effect demanded by the cosmological principle. For cosmological redshifts of $z<0.1$ the effects of spacetime expansion are minimal and observed redshifts dominated by the peculiar motions of the galaxies relative to one another that cause additional Doppler redshifts and blueshifts. In particular, Doppler redshift is bound by special relativity; thus $v>c$ is impossible while, in contrast, $v>c$ is possible for cosmological redshift because the space which separates the objects (e.g., a quasar from the Earth) can expand faster than the speed of light. The cosmological redshift, occurs between two clocks that are at different radii and both at rest with respect to the black hole or other center of gravitational attraction. Visible light with the longest period is red. The remote observer see light emitted by the close-in clock to be redder -that is of longer period- than it was at the point of emission. A photon moves through the spacetime, its wavelength is influenced by the expansion of the universe, as if the photon being attached to the expanding fabric spacetime. The cosmological (gravitational) redshift is a consequence of the changing size of the universe; it is not related to velocity at all. The gravitational redshift in curved expanding spacetime is a generalization of the Doppler shift in flat spacetime to curved expanding spacetime, is the reddening of light from distant galaxies as the universe expands. In the widely accepted cosmological model based on General relativity, redshift is mainly a result of the expansion of space: this means that the farther away a galaxy is from us, the more the space has expanded in the time since the light left that galaxy, so the more the light has been stretched, the more redshifted the light is, and so the faster it appears to be moving away from us. Hubble's law follows in part from the Copernican principle. Light waves become stretched in route between the time they were emitted long ago, and the time they are detected by us today. It is tempting to refer to cosmological redshifts as Doppler shifts. By referring to cosmological redshifts as Doppler shifts, we are insisting that our Newtonian intuition about motion still applies without significant change to the cosmological arena. A result of this thinking is that quasars now being detected at redshifts of $z=4.0$ would have to be interpreted as traveling a speeds of more than $V=z x$ $c$ or 4 times the speed of light. This is, of course, quite absurd, because we all know that no physical object may travel 
faster than the speed of light. To avoid such apparently nonsensical speeds, many popularizers use the special relativistic Doppler formula to show that quasars are really not moving faster than light. The argument being that for large velocities, special relativity replaces Newtonian physics as the correct framework for interpreting the world. By using a special relativistic velocity addition formula the quasar we just discussed has a velocity of 92 percent the speed of light. Although we now have a feeling that Reason has returned to our description of the universe, in fact, we have only replaced one incomplete explanation for another. The calculation of the quasar's speed now presupposes that special relativity (a theory of flat spacetime) is applicable even at cosmological scales where general relativity predicts that spacetime curvature becomes important. The special relativistic Doppler formula is introduced to show how quasars are moving slower than the speed of light! It is also common for popularizers of cosmology to describe how 'space itself stretches' yet continue to describe the expansion of the universe as motion governed by the restrictions of special relativity. By adopting general relativity as the proper guide, such contradictions are eliminated. General relativity leads us to several powerful conclusions about our cosmos:[8]

1) Special relativity is inapplicable for describing the larger universe;

2) The concepts of distance and motion are not absolutely defined and

3) Preexisting spacetime is undefined.

General relativity must replace special relativity in cosmology because it denies a special role to observers moving at constant velocity, extending special relativity into the arena of accelerated observers. It also denies a special significance to special relativity's flat spacetime by relegating it to only a microscopic domain within a larger geometric possibility. Just as Newtonian physics gave way to special relativity for describing high speed motion, so too does special relativity give way to general relativity. This means that the special relativistic Doppler formula should not, in fact cannot, be used to quantify the velocity of distant quasars. We have no choice in this matter if we want to maintain the logical integrity of both theories. The instantaneous physical distance is not itself observable. Cosmological 'motion' cannot be directly observed. It can only be inferred from observations of the cosmological redshift, which general relativity then tells us that the universe is expanding. One of the most remarkable discoveries in twentieth century astronomy was Hubble's (1929) observation that the redshifts of spectral lines in galaxies increase linearly with their distance. Hubble took this to show that the universe is expanding uniformly, and this effect can be given a straightforward qualitative explanation in the FLRW models. The FLRW models predict a change in frequency of light from distant objects that depends directly on scale factor $R(t)$. There is an approximately linear relationship between redshift and distance at small scales for all the FLRW models, and departures from linearity at larger scales can be used to measure spatial curvature. Locally the spacetime is flat. For distant objects, the imprint of the curvature is significant, where the spacetime does no longer remain flat. The redshifts of such distant objects increase according to the curvature of the hyperbolic spacetime. The cosmological (gravitational) redshift can be interpreted as a degree of the hyperbolicity of the curved spacetime. Hubble's law ( $\mathrm{V}_{\text {rec }}=\mathrm{HD}$ : recession velocity $=$ Hubble's constant $\times$ distance) describes the situation: farthest objects receding fastest. It didn't explain why? Hubble himself was not entirely happy with his distance-velocity formula, which decisively contributed to the inflationary model of the universe. In the paper, jointly with Tolman, he wrote "The possibility that the redshift may be due to some other cause connected with the long time or distance involved in the passage of light from nebulae to observer, should not be prematurely neglected."[22] "The Hubble velocity distance rule is an interesting example how two independently correct facts, i.e. the common Doppler shift and Hubble's experimental distance vs redshift law when "married" together resulted in an unfortunate conclusion. This happened because the only cause of redshift that Hubble was aware, was the common Doppler shift, and thus he obtained a distance-velocity plot" [9] " In a general setting and from a logical point of view, the existence of relative velocity is a necessary but not sufficient condition to record a wavelength shift. In Euclidean geometry e.g. wavelength shift uniquely implies existence of a relative velocity while in hyperbolic geometry it does not have a unique implication. Thus while the existence of relative velocity always results in a wavelength shift, the presence of a shift may or may not imply the existence of a relative velocity. Euclidean geometry cannot induce changes in wavelength of electromagnetic radiation. The case of $K=0$. In Euclidean space geodesics do not deviate. This is the case of hyperbolic space. Geodesics deviate at an exponential rate" [9].

\section{Quasars Redshifts Don`t Exhibit Time Dilation}

The phenomenon of time dilation is a strange yet experimentally confirmed effect of relativity theory. One of its implications is that events occurring in distant parts of the universe should appear to occur more slowly than events located closer to 
us. For example, when observing supernovae, scientists have found that distant explosions seem to fade more slowly than the quickly-fading nearby supernovae. However, a new study has found that this doesn't seem to be the case - quasars, it seems, give off light pulses at the same rate no matter their distance from the Earth, without a hint of time dilation. Astronomer Mike Hawkins from the Royal Observatory in Edinburgh came to this conclusion after looking at nearly 900 quasars over periods of up to 28 years. When comparing the light patterns of quasars located about 6 billion light years from us and those located 10 billion light years away, he was surprised to find that the light signatures of the two samples were exactly the same. If these quasars were like the previously observed supernovae, an observer would expect to see longer, "stretched" timescales for the distant, "stretched" high-redshift quasars. But even though the distant quasars were more strongly redshifted than the closer quasars, there was no difference in the time it took the light to reach Earth. [10]

Quasars have redshifts variation not correlated with time dilation. The light signature of quasars located 6 billion light years from us and those 10 billion light years away were exactly the same, without a hint of time dilation. This quasar conundrum doesn't seem to have an obvious explanation. Thus the high redshifts of quasars may not necessarily represent their distances. Further, in some observations, the redshifts have been found to exhibit some periodicity in their distributions as represented by the Karlsson formula [11]. The periodicity further makes it difficult for the redshift to represent distance. $M$ Hawkins is very clear, his finding is that: the redshift of the Quasars do not exhibit time dilation. Moreover, he gave many suggestions: [12]

1. It means the quasars may be nearby, not as distant as their redshifts and the Hubble law would indicate.

2. The origin of all matter was not at the big bang but over time in a grand ongoing creation scenario.

3. The Universe is not expanding.

4. Several explanations are discussed, including the possibility that time dilation effects are exactly offset by an increase in timescale of variation associated with black hole growth, or that the variations are caused by microlensing in which case time dilation would not be expected. [12]

In April 2010, Marcus Chown wrote in an article entitled [13] "Time waits for no quasar-even though it should" for New Scientist online. The edifice of the big bang hangs on the interpretation that the quasar redshifts are cosmological. If they are not: it brings into question the origin of quasars, and, it means the quasars may be nearby, not as distant as their redshifts and the Hubble law would indicate. This latter idea is linked to the work of Halton Arp [14] and others that showed strong correlation between parent galaxies that have ejected quasars from their active cores. The origin of all matter was not at the big bang but over time in a grand ongoing creation scenario. Arp [14] believed quasars were ejected from the active hearts of parent galaxies and their redshifts were largely intrinsic, not distance related. Because most of the high redshift objects in the Universe are quasars, if their redshifts are due to cosmological expansion then they are good evidence for an expanding universe. If the quasar redshifts are not reliable as a distance indicator, as Arp's hypothesis of ejection of quasars from the active cores of relatively nearby galaxies suggests, then the conclusion that the universe is expanding can be avoided. Arp, in fact, believed in a static universe [14]. The Hubble law, determined from the redshifts of galaxies, for the past 80 years, has been used as strong evidence for an expanding universe. This claim is reviewed in light of the claimed lack of necessary evidence for time dilation in quasar and gamma-ray burst luminosity variations and other lines of evidence. It is concluded that the observations could be used to describe either a static universe (where the Hubble law results from some as-yet-unknown mechanism) or an expanding universe described by the standard cold dark matter model. In the latter case, size evolution of galaxies is necessary for agreement with observations. Yet the simple nonexpanding Euclidean universe fits most data with the least number of assumptions [15].

\section{Why all Quasars are Redshifted?}

Quasars are believed to be objects ejected from the centers of the Galaxies (or Black holes). Do all of them blow outwards in opposite direction to us in order all of them to agree with such high redshifts? Note that the motion of galaxies is random! While, even no one Quasar exhibits a blueshift! Moreover, according to their high redshift all of the Quasars are very distant away. But the universe is isotropic, so our position is not preferred. Hence why we didn't observe any Quasar nearby? According to the isotropy, a distant observer should observed the Quasars very distant with respect to him, that is they should be nearby to us, a contradiction. According to Hubble's law, if the object is bright then its nearby and the distant objects are faint. The Quasars are very bright, so why they shouldn't be nearby? Why we just accept one part from Hubble's law, that is: the high redshift of the Quasar indicates that its distant and ignored the other part, that is: the brightness of the 
Quasar indicates they are nearby? Finally, why our Galaxy and many other nearby Galaxies didn't eject Quasars from their centers? Why this job is exclusive for distant Galaxies? Because our Galaxy and many others nearby Galaxies are inactive, said astronomers. Why they are the inactive among the active distant Galaxies? It is clear such a paradigm is not satisfactory and insufficient, it depends on many unjustified reasons, many contradictions and inconsistent. The paradigm must be reconsidered and readjusted. The bright the Quasar, the high it's redshift and the distant it is. The bright the Galaxy the low redshift, the nearby it is. Brightest Galaxies associated with brightest Quasars, but faint Galaxies not. So, if the Quasars agree in their brightness they disagree with their redshifts. Yes, the scenario concerning the Quasars no more than speculations and guesses to fabricate suitable explanations to fit current observations. The problem relies on the similarity of the cosmological redshift to the Doppler redshift that both of them cause recession speed. The first by the expansion of the spacetime and the other by receding within the spacetime. If the high redshift of the Quasar is due to the cosmological redshift of the expanding spacetime, why it shouldn't agree and coincide with the redshift of the hosting Galaxy. The cosmological redshift must be interpreted in a different way, as I do, as manifests the curvature of the hyperbolic spacetime. Astronomers have found many galaxy pairs and galaxy groups in which the members are evidently close to each other - even interacting — yet have redshifts that are radically at odds! Their redshifts don't make sense: If two galaxies are roughly in the same place then their measured redshifts should agree with each other, since redshift is supposed to be a measure of their distance (although the redshift may include a relatively minor Doppler component due to local motion). The observational fact that they don't is considered anomalous. The mystery is in the cause, and also why some of the anomalies are so extreme. "Locally the spacetime is flat, where special relativity together with its Doppler redshift dominates to measure peculiar velocities, there is no cosmological redshift in this case. For distant objects the spacetime is hyperbolic where the cosmological redshift manifests the curvature" [16]. For example, observations tell us that space within galaxies, which are rather diffuse objects, do not expand. Thus, where is the "border line" in space which divides expanding space from non expanding space?

Arp (1987); Ratcli (2010) [17] have argued that there is strong observational evidence for anomalous redshifts between quasars and galaxies. Typically there is a quasar very close to a galaxy with a material bridge or other evidence that suggests that they are associated. Two galaxies within our Local Group, including Andromeda, and a few galaxies in the Virgo Cluster display blueshifts and so are moving toward us, but these results from their local motion (peculiar velocity). Why are nearby galaxies exhibit blue-shift? Because their peculiar velocities are greater than their recession velocities! How could we compare between two incompatible concepts, the cosmological redshift and the Doppler redshift? What is the resultant crossbred-shift? The answer would be more convenient if we said: Locally the spacetime is flat through which the curvature is negligible (no cosmological redshift), the random peculiar velocity dominates. For distant objects the spacetime is hyperbolic, where the cosmological redshift manifests the curvature. If cosmological redshift has nothing to do with the Doppler effect, how do we know that galaxies that are very far away are also receding from us? How to compare between two unrelated concepts, the Doppler redshift and the cosmological redshift? Andromeda galaxy is blueshifted because it's sufficiently nearby where the spacetime is approximately flat and special relativity dominates. Its blueshifted according to the Doppler Effect in flat spacetime. Andromeda one of about 100 blueshifted galaxies that we observe. Andromeda has a "blueshift". It has a negative recessional velocity of roughly $-300 \mathrm{~km} / \mathrm{s}$ Andromeda's tangential or sideways velocity with respect to the Milky Way is relatively much smaller than the approaching velocity. Locally the spacetime is flat no cosmological redshift, their blueshifts is just due to the Doppler Effect. What causes the peculiar velocity of the galaxy? Is it a free fall or something else? As you probably know, we interpret the redshifts of galaxies to mean that the universe is expanding. So if you could staple the galaxies to the 'fabric' of space, all of them would appear to be moving away from us -the farther away they are, the faster! Why? This is cheating! According to the isotropy principle our position is not preferred. Conversely the farther observer would see our nearby objects recede faster with respect to him than his nearby objects! A contradiction.

\section{The Distance Horizon in a Flat Universe}

Consider a photon moving along a radial trajectory in a flat universe. A radial null path obeys [18] $o=d s^{2}=-d t^{2}+a^{2}(t) d r^{2}$

$$
r=\text { Ò }_{t_{e}}^{t_{0}} \frac{d t}{a(t)}
$$


For matter dominated component of energy :

$R \mu t^{2 / 3}$

Hubble constant now (at $\left.t_{0}\right)$ is

$$
H_{\mathrm{o}}=H\left(t_{\mathrm{o}}\right)=\frac{\dot{a}\left(t_{\mathrm{o}}\right)}{a\left(t_{\mathrm{o}}\right)}
$$

So the age of the universe now is

$$
\begin{aligned}
& t_{0}=\frac{2}{3 H_{0}} \\
& \because H_{0}=72(\mathrm{~km} / \mathrm{s}) / M p c \\
& \backslash t_{0}=\frac{2}{3 H_{0}}=9 \mathrm{Gyr}
\end{aligned}
$$

which is inconsistent compared to the age of the oldest stars whose age is about $12 \mathrm{Gyr}$ in our galaxy. Equations due to the flat universe doesn't fit the data. The physical distance to the horizon-in flat FRW model- at the time of observations is

$$
\begin{aligned}
& d_{H}(t)=a(t) r_{H}=a(t){ }_{0}^{t} \frac{d t \phi}{a(t \phi)} \\
& \because a(t) \mu t^{2 / 3} \\
& \backslash d_{H}(t)=a(t) r_{H}=t^{2 / 3}{ }_{0}^{t} \frac{d t \phi}{t e^{2 / 3}}=3 t
\end{aligned}
$$

The present horizon size of a matter dominated flat universe

$$
\begin{aligned}
& d_{\text {horizon }}\left(t_{0}\right)=3 t_{0}=3 \underset{\mathrm{e}}{\stackrel{\not 2}{\rightleftarrows}} t_{H} \frac{\ddot{\mathrm{o}}}{\dot{\bar{\Phi}}} \\
& d_{\text {horizon }}\left(t_{0}\right)=2 t_{H} \gg 8 \mathrm{Gpc}
\end{aligned}
$$

The discrepancy between this number and the $14 \mathrm{Gpc}$ (observed radius in principle) is due to the presence of the significant vacuum energy (dark energy). Note that Hubble radius $H_{\mathrm{O}}^{-1}=4.2^{\prime} 10^{3} \mathrm{Mpc}$.

\section{The Distance Horizon is Infinite in a Hyperbolic Universe.}

"Because the Universe is expanding the distance to a galaxy is not very well defined. Because of this ambiguity, astronomers prefer to work in terms of a look-back time, which is simply how long ago an object emitted the radiation we see today. Astronomers talk frequently about redshifts and sometimes about look-back times, but they hardly ever talk of distances to high-redshift objects. The redshift is the only unambiguously measured quantity. Statements about derived quantities, such as distances and look-back times, all require that we make specific assumptions about how the universe has evolved with time. For nearby sources, the look-back time is numerically equal to the distance in light-years. However, for more distant objects, the look-back time and the present distance in light-years differ because of the expansion of the universe, and the divergence increase dramatically with increasing redshift" [19]. Comoving distance "is the distance 
between two points measured along a path defined at the present cosmological time. For objects moving with the Hubble flow, it is deemed to remain constant in time. The comoving distance from an observer to a distant object (e.g. galaxy) can be computed by the following formula:

$$
c=\dot{\omega}_{t_{e}}^{t_{0}} \frac{d t \phi}{a(t \phi)}
$$

where $a\left(t^{\prime}\right)$ is the scale factor, $t_{\mathrm{e}}$ is the time of emission of the photons detected by the observer, $t$ is the present time, and $c$ is the speed of light in vacuum. Despite being an integral over time, this does give the distance that would be measured by a hypothetical tape measure at fixed time $t$, i.e. the "proper distance" as defined below, divided by the scale factor $a(t)$ at that time. The isotropic homogeneous Robertson- Walker's line-element:

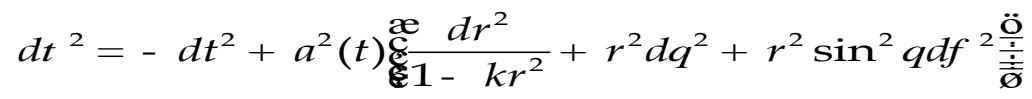

For the hyperbolic spacetime

$$
\begin{aligned}
& k=-1
\end{aligned}
$$

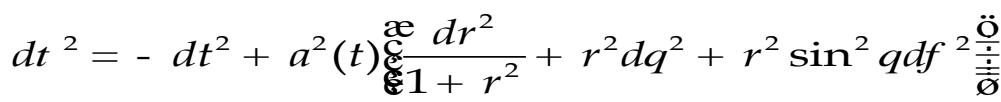

For radial null trajectory

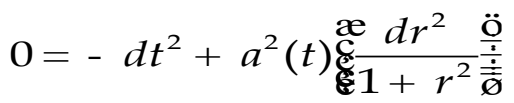

$$
\begin{aligned}
& \frac{d r}{\sqrt{1+r^{2}}}=\frac{d t}{a(t)}
\end{aligned}
$$

The physical radius to the horizon-in hyperbolic universe FRW model- at the time of observations is

$$
\mathrm{O}_{0}^{r} \frac{d r \phi}{\sqrt{1+r \phi^{2}}}=\mathbf{\mathrm { O }}_{0}^{t} \frac{d t \phi}{a(t \phi)}
$$

We have the scale factor in the hyperbolic universe

$$
a(t)=\sqrt{3 / 8 p r_{\mathrm{j}}} \sinh t \sqrt{8 p r_{\mathrm{j}} / 3}
$$

Substitute this scale factor in Eq.(6), we get

$$
\begin{aligned}
& \grave{\mathrm{O}}_{0}^{r} \frac{d r \phi}{\sqrt{1+r \phi^{2}}}=\grave{\mathrm{O}}_{0}^{t} \frac{d t \phi}{\sqrt{3 / 8 p r_{\mathrm{j}}} \sinh t \phi \sqrt{8 p r_{\mathrm{j}} / 3}} \\
& \grave{\mathrm{O}}_{0}^{r} \frac{d r \phi}{\sqrt{1+r \phi^{2}}}=\sqrt{8 p r_{\mathrm{j}} / 3}{\underset{\mathrm{O}}{\mathrm{O}}}_{0}^{t} \frac{d t \phi}{\sinh t \phi \sqrt{8 p r_{\mathrm{j}} / 3}} \\
& \mathrm{ò}_{0}^{r} \frac{d r \phi}{\sqrt{1+r \phi^{2}}}=\sqrt{8 p r_{\mathrm{j}} / 3} \stackrel{\mathrm{o}}{t}_{0}^{t} \operatorname{coseht} t \sqrt{8 p r_{\mathrm{j}} / 3} d t \phi
\end{aligned}
$$




$$
\begin{aligned}
& \sin h^{-1} r \phi_{0}^{r}=\left.\operatorname{coth}^{-1} \cosh t \phi \sqrt{8 p r_{\mathrm{j}} / 3}\right|_{0} ^{t} \\
& \backslash \sin h^{-1} r-\sin s h^{-1} 0 \\
& =\operatorname{coth}^{-1} \cosh t \sqrt{8 p r_{\mathrm{j}} / 3}-\operatorname{coth}^{-1} \cosh 0 \\
& \backslash \ln \left|r \pm \sqrt{1+r^{2}}\right|-\ln | \pm 1| \\
& =\frac{1}{2} \ln \left|\frac{\cosh t \sqrt{8 p r_{j} / 3}+1}{\cosh t \sqrt{8 p r_{j} / 3}-1}\right|-\frac{1}{2} \ln \left|\frac{\cosh 0+1}{\cosh 0-1}\right| \\
& \backslash \ln \left|\frac{\mid r \pm \sqrt{1+r^{2}}}{ \pm 1}\right| \\
& =\ln \left|\frac{\sqrt{\left(\cosh t \sqrt{8 p r_{\mathrm{j}} / 3}+1\right) /\left(\cosh t \sqrt{8 p r_{\mathrm{j}} / 3}-1\right)}}{\sqrt{(\cosh 0+1) /(\cosh 0-1)}}\right| \\
& \backslash r \pm \sqrt{1+r^{2}}=\frac{\sqrt{\left(\cosh t \sqrt{8 p r_{\mathrm{j}} / 3}+1\right) /\left(\cosh t \sqrt{8 p r_{\mathrm{j}} / 3}-1\right)}}{\sqrt{(2) /(0)}} \\
& \backslash r \pm \sqrt{1+r^{2}}=\frac{\sqrt{\left(\cosh t \sqrt{8 p r_{\mathrm{j}} / 3}+1\right) /\left(\cosh t \sqrt{8 p r_{\mathrm{j}} / 3}-1\right)}}{¥} \\
& \begin{array}{l}
\backslash r \pm \sqrt{1+r^{2}}=0 \\
\backslash r^{2}=1+r^{2} \\
r_{\text {norizon }}= \pm r^{2}
\end{array}
\end{aligned}
$$

The distance horizon in the hyperbolic universe is infinite, simply solves the horizon problem. [20]

\section{Conclusion}

1- The Hubble law, determined from the redshifts of galaxies, for the past 80 years, has been used as strong evidence for an expanding universe. This claim is reviewed in light of the claimed lack of necessary evidence for time dilation in quasar and gamma-ray burst luminosity variations and other lines of evidence. It is concluded that the observations could be used to describe either a static universe.

2-The cosmological redshift manifest the curvature : Hubble's law describes a uniformly expanding flat universe. Hubble's law doesn't explain why distant objects were receding fastest. There is an approximately linear relationship between redshift and distance at small scales for all the FLRW models, and departures from linearity at larger scales can be used to measure spatial curvature. Locally the spacetime is flat. For distant objects, the imprint of the curvature is significant, where the spacetime does no longer remain flat. The redshifts from such distant objects increase according to the increase in the curvature of the hyperbolic spacetime. The cosmological redshift can be interpreted as a degree of the hyperbolicity of the curved spacetime, not a distance indicator. Newton first law states that the body keeps moving with a uniform velocity in straight line. Similarly, the free fall of an object in a flat spacetime is uniform. An accelerated motion is described by a curve. For large structure, the curvature of the spacetime can't be ignored. The expansion of the universe is described by a hyperbolic curve. The distant objects- e.g. supernovae - were influenced under the curvature of the spacetime. They possess an accelerating free fall due to the curvature of the hyperbolic spacetime that manifests itself by the equation of 
the state $p=-r$, which is the property of the hyperbolic structure of the Universe. The apparent accelerated expansion is a consequence of a curved (hyperbolic) spacetime.

3- The distance horizon is infinite in a hyperbolic universe: answers the question: What does the universe expand into? The observable Universe is expanding into a pre-existing infinite distance horizon. This question is a forbidden and prohibited question in the Standard Big Bang Cosmology? The Big Bang theorists insist that the above question has no meaning, instead of saying that they have no answer or that it violates their entire paradigm.

4-The Big Bang's Pillars are refuted and disproved:

The expansion-of-space cosmic redshift.

We show that the cosmological redshift is no longer a distance indicator. The Cosmological Redshift just manifests the curvature.

\section{Singularity}

We show the manifold of the Universe is complete with nonsingular point.

The cosmic microwave background radiation (CMBR).

The problem with the 2nd Pillar is that the CMBR is simply the background temperature of our infinite Universe. It is merely ultra-distant starlight that has undergone extreme cosmic redshifting (light wave stretching). [21]

\section{Olber's Paradox}

Olber's Paradox is not a problem. Curvature redshift is sufficient to move distant starlight out of the visible band. Visible light from distant galaxies is shifted into the infrared where it is no longer seen [20].The Big Bang has lost its Pillars. It is no longer a viable hypothesis. A theory or hypothesis that has no supporting pillars is merely pseudo-science, at best. The Big Bang has been exposed for what it has always been, an elaborate misconceptional myth wrapped in pseudo-science a grand creation myth!

5- After all, in the hyperbolic spacetime a group of objects would grow apart even when not moving as their worldlines would be divergent; this we mean by the expansion of the hyperbolic spacetime.

\section{References}

[1] (PhysOrg.com) April 9, 2010 by Lisa Zyga : http://phys.org/news/2010-04-discovery-quasars-dont-dilationmystifies.htm|\#jiCp

A. Einstein, (1921): The Meaning of Relativity. Princeton University Press (2005). PP. 117-18.

[2] Arp et al. 1990, Burbidge \& Napier. 2001.

[3] Astrophys. J. 84, 517H (1936).

[4] Chaisson. E, McMillan. S:Astronomy Today $6^{\text {th }}$ ed. pp 666-7.

[5] Chown, M., Time waits for no quasar - even though it should, NewScientist.com, April 7, 2010

[6] Conrad Ranzan, The dynamic steady state universe. PHYSICS ESSAYS 27, 2 (2014). http://dx.doi.org/10.4006/0836-1398-27.2.286]

[7] Fig (1) Earthsky.org, What is dark energy.

[8] http://www.haltonarp.com/articles.

[9] http://www.universe-galaxies-stars.com/Redshift.html (access day 9/12/2016).

[10] https://plato.stanford.edu/entries/spacetime-singularities (access day 9/12/2016). 
[11] J. Georg von Brzeski, Epansion of the niverse - mistake of Edwin Hubble? Cosmological redshift and related electromagnetic phenomena in static Lobachevskian (Hyperbolic) Universe Actaphysica POLONICA B Vol. 39 (2008). No. 6.

[12] J.G. Hartnett, Is the Universe really expanding? rXiv:1107.2485v2 [physics.gen-ph] 19 Nov 2011.

[13] James B. Hartle(2003): Gravity An Introduction To Einstein's General Relativity. Addison Wesley. P 409

[14] James B. Hartle(2003): Gravity An Introduction To Einstein's General Relativity. Addison Wesley. P 11

[15] MRS Hawkins, On time dilation in quasar light curves, Mon Not Roy Astron Soc, 405 (2010) 1940-6.

[16] Ratcli_e, H., (2010). Anomalous Redshift Data and the Myth of Cosmological Distance. /joc, 413, 109.

[17] Salah A. Mabkhout (2012), The hyperbolic geometry of the universe and the wedding of general relativity theory to quantum theory. Physics Essays: March 2012, Vol. 25, No. 1, pp. 112-118.

[18] Salah A. Mabkhout (2013) The Big Bang hyperbolic universe neither needs inflation nor dark matter and dark energy. Physics Essays: September 2013, Vol. 26, No. 3, pp. 422-429.

[19] Salah A. Mabkhout, The Cosmological Redshift Manifests the Curvature and Interpreted as a Degree of Hyperbolicity of the Spacetime. Journal for Foundations and Applications of Physics, vol. 3, No. 1 (2016). (sciencefront.org).

[20] Salah A. Mabkhout, The Infinite Distance Horizon and the Hyperbolic Inflation in the Hyperbolic Universe. Elixir Space Sci. 94 (2016) 40120-40137

[21] Sean M. Carroll (2004), Spacetime and Geometry. Addison Wesley. p : 347.

[22] Sten Odenwald and Rick Fienberg: Galaxy Redshift Reconsidered. Cecelia.physics.indiana.edu .http://www.astronomycafe.net (access day 11/19/2015) 